\title{
PATRONES DE GÉNERO EN LA PRÁCTICA DEL TRABAJO INFANTIL: EL CASO DE LAS NIÑAS JORNALERAS EN LA REGIÓN FLORÍCOLA DEL ESTADO DE MÉXICO*
}

\author{
SARAI MIRANDA JUÁREZ $Z^{* *} \&$ MARIELA MACRI ${ }^{* * *}$ \\ UNIVERSIDAD DE BUENOS AIRES (ARGENTINA)
}

Recibido/ Received/ Recebido: 07/04/2014 - Aceptado/ Accepted / Aprovado: 12/07/2014

\begin{abstract}
Resumen
El presente artículo tiene como objetivo general conocer y analizar las principales manifestaciones laborales que subyacen en las preconcepciones del género y que impactan en la práctica del trabajo de las niñas jornaleras en la producción de flores. La metodología utilizada es de corte cualitativo que incluyó entrevistas semiestructuradas como parte del trabajo etnográfico. Las conclusiones que se hallaron se relacionan con el importante lugar de la condición de género en la diferenciación entre niñas y niños trabajadores.
\end{abstract}

Palabras clave: Trabajo infantil, Niñas, Género, Floricultura, México.

\section{GENDER PATTERNS IN THE PRACTICE OF CHILD LABOR: THE CASE OF WORKING GIRLS ON MEXICO STATE IN THE REGION OF FLOWERS PRODUCTION}

\begin{abstract}
The objective of this study was to know and to analyze the main labor manifestations that underlay the gender preconceptions that affect the practices of working girls in the flower production. As part of the ethnographic work, semi-structured interviews were conducted. The findings show that the gender condition plays an important role in the differences between working girls and boys.
\end{abstract}

Keywords: Child labor, Girls, Gender, Floriculture, Mexico.

Artículo de investigación derivado de la estancia post doctoral de Sarai Miranda Juárez en el Instituto de Investigaciones Gino Germani de la Universidad de Buenos Aires (Argentina).

** Doctora en Estudios de Población, actualmente es investigadora invitada en modalidad de estancia post doctoral en el Instituto de Investigaciones Gino Germani de la Universidad de Buenos Aires. Correo electrónico: saraimirandaj@gmail.com. Tel: (+54 11) 4508.3815. Dirección postal: Pte J. E. Uriburu 950, 6to. (C1114AAD) Ciudad Autónoma de Buenos Aires.

***: Doctora en Educación, actualmente dirige el Grupo de Investigación sobre Infancia, Adolescencia y Juventud del Instituto de Investigaciones Sociales Gino Germani de la Universidad de Buenos Aires. Correo electrónico: m_macri@fibertel.com.ar. Tel: (+54 11) 4508.3815. Dirección postal: Pte J. E. Uriburu 950, 6to. (C1114AAD) Ciudad Autónoma de Buenos Aires. 


\title{
PADRÕES DE GÊNERO NA PRÁTICA DO TRABALHO INFANTIL: O CASO DAS MENINAS DIARISTAS NA REGIÃO FLORÍCOLA DO ESTADO DE MÉXICO
}

\begin{abstract}
Resumo
O presente artigo tem como objetivo geral conhecer e analisar as principais manifestações laborais que subjazem nas preconcepções de gênero e que impactam na prática do trabalho das meninas diaristas na produção de flores. A metodologia utilizada é de corte qualitativo que incluiu entrevistas semiestruturadas como parte do trabalho etnográfico. As conclusões encontradas se relacionam com o importante lugar da condição de gênero na diferenciação entre meninas e meninos trabalhadores. Palavras chave: Trabalho infantil, Meninas, Gênero, Floricultura, México.

Miranda, S. \& Macri, M. (2015). Patrones de género en la práctica del trabajo infantil: el caso de las niñas jornaleras en la región florícola del Estado de México En: Revista de la Facultad de Ciencias Económicas de la Universidad Militar Nueva Granada. rev.fac.cienc.econ, XXIII (1).
\end{abstract}

JEL: J01, J16, J43.

\section{Introducción}

Al sur del Estado de México, a solo 45 minutos de la capital de esta entidad se encuentra la mayor extensión territorial en México dedicada al cultivo de flores de corte. La región florícola se ubica en el distrito agrícola de Coatepec Harinas y comprende los municipios de Villa Guerrero, Coatepec Harinas, Tenancingo, Zumpahuacán, Ixtapan de la Sal, Tonático, Malinalco, Ocuilan, Texcaltitlán, Almoloya de Alquisiras, Sultepec y Zacualpan. Transitar a través de la autopista principal México-Ixtapan de la Sal resulta en una experiencia visual de fuertes contrastes. El paisaje es un extenso valle repleto de árboles de bosques de coníferas, los altos cedros y fresnos comparten espacios con las amplias arboledas verdes de aguacates y duraznos.

Por sus características climatológicas y geográficas esta región cuenta con una amplia diversidad de flora y fauna que difícilmente pasa inadvertida desde la carretera. Ahí mismo, pero por debajo de ese suelo fértil, atraviesan un sin número de corrientes de agua dulce y limpia que provienen en su mayoría del volcán Xinantécatl, comúnmente conocido como el Nevado de Toluca.

De entre la tupida arboleda se asoman también grandes estructuras metálicas cubiertas de plásticos blancos que alojan en su interior una vasta gama de flores de corte producidas bajo estos invernaderos con el objetivo de controlar su proceso de crecimiento. Igualmente hacen parte del paisaje túneles cubiertos de nailon blanco construidos de forma mucho más improvisada, pero que funcionan también para la producción controlada de todo tipo de flores de corte y follajes.

Este extenso paisaje forestal en el que se han intercalado rectángulos blancos plastificados contiene en su interior una compleja dinámica social asociada a la actividad florícola comercial. El mercado de trabajo local se compone de familias enteras dispuestas a emplearse en las temporadas de siembra, corte y pos cosecha de flores. Las estimaciones realizadas calculan que tan sólo en el municipio de Villa Guerrero de forma permanente se emplean aproximadamente siete empleados por hectárea cultivada, es decir, 84 mil personas empleadas en actividades relacionadas con la producción floral, cantidad que en temporadas de alta producción llega a triplicarse.

La actividad florícola de esta región al igual que otros cultivos modernos y de vanguardia como las hortalizas y las frutas, es intensivo en el uso de recursos tales como la energía, la innovación tecnológica, el capital, pero sobre todo en fuerza de trabajo de 
carácter temporal. Ello implica que como todo modelo de intensificación en el agro se acompañe de los problemas tradicionales de los mercados de trabajo así como la emergencia de nuevas dinámicas laborales a su alrededor, en los que la tendencia se dirige al deterioro de las condiciones de contratación y uso del factor trabajo a través de la profundización de modalidades como trabajo a destajo y agricultura por contrato. Una constante en el espacio agrícola actual es la mercantilización y flexibilización de la mano de obra campesina, lo que ha llevado a la diversificación del trabajo asalariado $y$, sobre todo, a la constante presencia de la población femenina e infantil empleada en el agro (Seffó, 2005).

Como afirma Morett \& Cosío $(2004,8)$ esta realidad tiene como base esencial el principio de las ventajas comparativas, que consiste básicamente en el máximo aprovechamiento de la fuerza de trabajo, "de ahí que las actividades más rentables en la actualidad sean, (...) grandes captadoras de mano de obra, lo cual implica que se sustentan en la ventaja de los bajos salarios rurales que les pueden permitir tener costos de producción similares a los de los países desarrollados".

Así, en la región florícola del Estado de México durante los tiempos de alta producción a muy tempranas horas del día es común encontrar grupos de familias completas esperando a pie de carretera en los principales paraderos de la autopista México-Ixtapan de la Sal para realizar tratos con los enganchadores o intermediarios a fin de ser empleados en alguna empresa floricultora.

Las niñas jornaleras representan una parte importante del contingente de trabajadores dispuestos a laborar bajo condiciones precarias y flexibles en un sector productivo con altas tasas de ganancias. En palabras propias del gerente de Fertilizantes Koor Industries LTD la floricultura en Villa Guerrero "es un negociazo, porque es netamente de exportación y se calcula en dólares" (Revista Expansión, 1998, 310).

Además de un modelo flexible de producción y organización de la fuerza de trabajo, el mantenimiento y crecimiento de la producción de flores de la región ha encontrado terreno fértil en las precarias condi- ciones de vida de la población campesina que poco a poco ha visto deteriorar su capacidad productiva $y$ competitiva.

Las estimaciones oficiales han puesto en evidencia que los niveles de pobreza rural superan por mucho a los niveles de pobreza en las áreas urbanas, "en las zonas rurales, el porcentaje de la población que vivía en pobreza en 2010 fue de 65,1 por ciento, frente a 40,6 por ciento en las zonas urbanas" (CONEVAL, 2012).

Los diagnósticos realizados muestran que en el ámbito rural las carencias sociales tuvieron incidencias de población que duplicaron, y en algunos casos quintuplicaron a las observadas en las zonas urbanas. El caso del acceso a la seguridad social y la cobertura de infraestructura básica para las viviendas con ejemplos de ello. En las zonas rurales la carencia de seguridad social fue de $81,9 \%$ de la población mientras que $54,3 \%$ para las zonas urbanas (CONEVAL, 2012).

Al mismo tiempo se observa que una serie de nuevas contradicciones permean hoy en día el funcionamiento del sector agropecuario en México. Tales contradicciones se materializan en una situación de desigualdad entre grandes y pequeños productores pues se ha favorecido fundamentalmente a la agricultura con mayor capitalización, es decir a los productores con mayores recursos mientras que se ha desplazado a las principales actividades económicas de las unidades familiares y campesinas, debido a que les es imposible competir en los mercados internacionales, con lo que se han visto en la necesidad de buscar estrategias para diversificar sus opciones de ingresos encontrando nuevas fuentes de sobrevivencia en fenómenos como la migración y la incorporación al trabajo asalariado de más miembros de la familia en las actividades agroindustriales más dinámicas tales como la floricultura comercial.

En materia de política económica hacia el campo mexicano, el impulso a los sectores agro exportadores modernos ha venido aparejado por varios cambios fundamentales en el sector agrícola mexicano y en la economía en general. Por un lado se presenta una disminución de producción de alimentos que 
ha generado mayor dependencia alimentaria. En la búsqueda de mercados externos y de generación de divisas, la política estatal hacia el agro se ha convertido en una estrategia poderosa para hacer uso de los recursos públicos.

Antes de la década de 1980, la política económica hacia el campo era de índole universal y enfocada hacia los granos básicos y la producción agroalimentaria básica para dar insumos al sector industrial; hoy ha cambiado, se dirige mayoritariamente hacia los productores que demuestren que pueden generar divisas; es decir, grandes productores capitalizados (Seffó, 2005).

La alta concentración de capitales, el achicamiento del Estado y su retracción en sus funciones redistributivas -salud, educación-, la reestructuración del mercado de trabajo desempleo, subempleo, precarización- y la caída del ingreso han aumentado las precarias condiciones de vida de vastos sectores sociales y especialmente en aquellos que históricamente eran pobres -pobres estructurales- (Lezcano, 2002).

Un fenómeno social que no es nuevo en el sector agrícola mexicano es el trabajo infantil, dado que tradicionalmente la estrategia de las familias rurales ha sido el trabajo de todos los miembros del hogar incluyendo a las niñas y los niños. La diferencia sustancial es que en la actualidad los niños y las niñas se emplean en un contexto de creciente competitividad entre países y en actividades que requieren incorporar estrategias para posicionarse en los mercados internacionales. Pero además la producción intensiva de frutas, flores y hortalizas frescas se sitúa en los sectores menos regulados de la política agrícola común internacional, y este sector está sometido de manera más fuerte a un capitalismo salvaje (Seffó, 2005).

De acuerdo con datos del último Módulo de Trabajo Infantil de las Encuestas Nacionales de Ocupación y Empleo, se estima que para el año 2011, el grupo de niños y niñas de 5 a 17 años que trabajan es de 3 millones y es justamente en el sector agrícola donde se ocupan la mayor parte de ellos, aproximadamente un millón en total, de los cuales $30 \%$ son niñas jornaleras (INEGI, 2012).
En la zona florícola del sur del Estado de México, las niñas jornaleras son el rostro oculto del mercado de trabajo local. Lejos de ser minoría como se esperaría a partir de las tendencias estadísticas nacionales, en la producción local de flores de corte para exportación las niñas jornaleras son un grupo mayoritario, que excede incluso a los niños varones, lo que da cuenta de la invisibilidad de las niñas jornaleras en las estadísticas nacionales pero además de la constante feminización de los mercados de trabajo rurales, caracterizados por la flexibilidad y la precariedad.

Como cualquier otro grupo poblacional, las niñas jornaleras empleadas en la floricultura de corte, requieren ser identificadas y volverse visibles frente a las políticas públicas, pues la sociedad y el Estado deben garantizar el respeto a los derechos más fundamentales de los niños y las niñas. En este sentido el objetivo general de este artículo es conocer y analizar las principales manifestaciones laborales que subyacen en las preconcepciones del género y que impactan en la práctica del trabajo de las niñas jornaleras en la producción de flores.

La estrategia metodológica que se utiliza para el logro de este objetivo es de corte cualitativo, utilizando información obtenida mediante entrevistas semiestructuradas y observación participante, ya que este artículo es el resultado de un trabajo de investigación más amplio en el que se realizó trabajo entnográfico en el contexto laboral de la región florícola del sur del Estado de México y se obtuvieron registros sobre las principales características laborales de las niñas jornaleras. El trabajo cualitativo se llevó a cabo entre los meses de enero y mayo de 2010, que son los meses de mayor demanda de fuerza de trabajo debido a la conmemoración de diversas fechas tales como San Valentín en febrero y el Día de las Madres en mayo.

La información que aquí se presenta fue recogida durante el trabajo de campo que se realizó en tres etapas: pre cosecha, corte y post cosecha, durante el ciclo primavera verano del 2010 en la zona florícola. Asistiendo a las empresas exportadoras, entrevistando a actores clave como empresarios, encargados, pequeños productores y representantes de las principales asociaciones de productores a nivel local y visitando las escuelas primarias de cinco localidades 
donde se realizaron grupos de discusión entre los niños y niñas que trabajan en la floricultura y se obtuvieron dibujos vinculados con la realidad laboral de estos niños y niñas.

\section{La floricultura y la feminización del mercado de trabajo}

El contexto de análisis desde el que se aborda la problemática de las niñas jornaleras se inscribe en un proceso de transformación agrícola local en el que convive un importante número de pequeños productores de subsistencia con pocas pero grandes empresas de capital multinacional.

Es en la década de los ochenta cuando comienza a consolidarse más claramente el lugar de la agricultura florícola en el sur del Estado de México, no obstante, la situación actual es el resultado de un proceso histórico en el que se establecen las bases para que esta región esté articulada productivamente a escala global. En términos generales se pueden diferenciar tres etapas. Una primera etapa, que abarca desde mediados del siglo XIX hasta principios del $\mathrm{XX}$, se sustenta en la preeminencia de la propiedad privada conocida como latifundismo que en México se difundió bajo la figura de la hacienda, esta etapa se disolvió parcialmente debido al proceso revolucionario que buscó repartir las grandes extensiones de tierra, y que se rebeló contra el latifundismo como sistema dominante. En esta primera etapa el sur del Estado de México se consolidó en un modelo dual en el que convivieron dos tipos de agricultura, por un lado una agricultura de tipo extensiva, basada en una abundante y barata fuerza de trabajo y especializada en actividades como el aguacate y los duraznos, y por otro lado posterior al proceso revolucionario y la aplicación de la reforma agraria, un modelo de agricultura fundamentado en la repartición de la tierra en ejidos parcelados, dedicados a cultivos más tradicionales como el maíz, la papa y el chicharo (Castro, 2003).

Una segunda fase, inicia en la década de los cincuenta y se desarrolla fundamentalmente durante las dos décadas que le siguen, esta etapa se caracteriza por la intensificación de las relaciones capitalistas de producción y la industrialización de la agricultura bajo el modelo económico de Sustitución de Importaciones a la par de lo que se denominó Revolución Verde (Rubio, 2002).

Esta etapa fue considerada como un proceso de modernización caracterizado por la creciente integración y supeditación de la agricultura a la industria, lo que supuso un papel significativo atribuido al sector agrícola como proveedor de insumos baratos al sector industrial, en el que los cultivos tradicionales cobraron mayor importancia, maíz, caña de azúcar, legumbres y verduras (Rubio, 2002).

Así, la agricultura del sur del Estado de México respondía a las exigencias de este modelo de desarrollo, suministraba materias primas que exigía la industria, y abastecía parte de la creciente demanda para el consumo de alimentos de la población urbana, aplicando con ello un proceso de industrialización de la agricultura sobre todo en materia de semillas mejoradas, abonos químicos, plaguicidas, fertilizantes, pero al mismo tiempo conviviendo con unidades económicas menos capitalizadas con producción de subsistencia (Aguado, 1998).

La tercera etapa es el contexto más inmediato en el que nace y se expande la floricultura de corte intensiva bajo invernadero en la región. Como muchas otras regiones del país, la región sur del Estado de México se vio inmersa en los efectos de la crisis internacional de los años setenta que obligó a las economías periféricas a cambiar su modelo productivo y de desarrollo, de ser economías basadas en el modelo de Sustitución de Importaciones a economías abiertas. Esta nueva política tuvo sus efectos en el sector agrícola con una reestructuración vinculada a un modelo global en el que se reformula el espacio agrícola mediante procesos de especialización regional y concentración del capital (Castro, 2003).

Es en este contexto en el que se sitúa el conocido boom de la floricultura, que fue favorecido por la liberalización de los mercados en pro de los grandes productores capitalizados y a costa de las economías campesinas de subsistencia. En este nuevo modelo se privilegia la agricultura con procesos tecnológicos de punta que se complementa con la gran distribución comercial, en el que priman sobre todo los 
criterios de rentabilidad económica por encima de las necesidades alimentarias y laborales de la población (Orozco, 2007).

Las más recientes mediciones indican que el cultivo de flores de corte en la zona sur del Estado de México se extiende en una superficie sembrada promedio de poco más de once mil hectáreas, esta superficie representa $0,6 \%$ del total de la superficie agrícola estatal y $86 \%$ de la superficie total dedicada a la floricultura nacional. Aporta $80 \%$ de las flores que exporta el país, y la comercialización de flores genera una derrama económica anual de 5.995 millones de pesos (617 millones de pesos sólo durante la temporada del Día de Muertos), ganancias que representan más de la cuarta parte de la derrama económica que genera el campo mexiquense (30\%) (Orozco, 2007).

Esta actividad ha sido impulsada por varios factores, entre ellos la introducción de la tecnología más avanzada. La investigación en biogenética aplicada a la producción de flores ha permitido una extensa gama de colores y matices tan exóticos como la mítica rosa azul, así mismo, ha generado un mayor periodo de duración desde que se corta el tallo hasta que permanece en un jarrón decorativo, lo que se traduce en mayor competitividad (Lara, 1998).

Igualmente, se empalman otras dimensiones que agregan ventajas al desarrollo de la floricultura como las condiciones climáticas, la disponibilidad de agua, el tipo de suelos de esta zona y las fuertes inversiones estatales en infraestructura carretera y de transporte que se han presentado en las tres últimas décadas.

Sin duda, un elemento fundamental para comprender el desarrollo y crecimiento de la producción de flores de corte bajo invernadero es la existencia de suficiente fuerza de trabajo local dispuesta a emplearse de forma temporal y bajo condiciones flexibles y precarias.

Al respecto cabe señalar que la zona florícola al igual que muchas otras zonas agrícolas del país experimentó un proceso de redistribución de tierras a partir de 1992 cuando se dio la modificación al Artículo 27 Constitucional en el que se aprobó la privatización de la tierra de tipo ejidal y comunal, con lo que se abrió la puerta a la burguesía local para obtener mayores extensiones de tierra con el propósito de ampliar el cultivo de la flor (Aguado, 1998). Paralelamente, se presenta la existencia de un sinnúmero de pequeños productores de subsistencia que responden a la estructura de agricultura familiar y que deben complementar sus ingresos incorporando al mayor número de miembros de la familia como trabajadores asalariados de los grandes floricultores.

Así, la floricultura de corte bajo invernadero, se desarrolla en el contexto de un modelo agrícola que como bien afirma Reigada $(2011,67)$ se caracteriza:

\section{“(...) por una mayor internacionalización y competitividad de los mercados; el peso que adquieren las corporaciones transnaciona- les, y especialmente las grandes cadenas de distribución; los avances en electrónica, microinformática, biotecnología y biogené- tica; la descentralización de las estructuras empresariales y una nueva organización espacial de la producción; y, junto a ello, la implantación de sistemas de producción flexibles que permitan superar la crisis del modelo fordista y ajustarse a la nueva nor- ma de consumo. La creciente demanda de productos 'en fresco' $y$ 'de primor' por par- te de las clases acomodadas y el aumento de mercancías que en otros tiempos eran consideradas exóticas, refleja el cambio en la naturaleza de la mercancía".}

Un aspecto de suma importancia tiene que ver con la intervención del Estado en el impulso de este tipo de actividades productivas. A pesar del presunto achicamiento estatal que se dio con las Reformas Estructurales aplicadas al sector agrícola mexicano, éste se ha encargado de dar apoyo con infraestructura y crédito a los productores más capitalizados.

En las pautas para el apoyo a las exportaciones de flores se formularon una serie de condiciones que deben cumplirse a fin de obtener algún apoyo financiero, de capacitación o logístico por parte del gobierno estatal y federal. Entre los requisitos están por ejemplo garantizar la calidad uniforme del producto y mantener una vida útil más larga, aplicando 
formas específicas de corte de tallo e incorporación de soluciones nutritivas; el empaque debe hacerse a granel o por conteo -manojos, ramilletes y tallos individuales-; el embalaje se deber realizar de acuerdo con la especie de flor -plancha de fibra, papel de seda, soluciones preservativas- (BANCOMEXT, 2001). Del mismo modo deben cumplirse normas ambientales y un certificado fitosanitario, sin embargo no se indica nada respecto a la gestión y organización de la fuerza de trabajo, en los manuales del exportador no aparece una sola línea que hable sobre el respeto a la legislación laboral o el manejo ético de los recursos humanos.

En este escenario, debe tomarse en cuenta que los cultivos modernos como las frutas, hortalizas y flores asisten a un proceso creciente de feminización del trabajo. Varios autores han documentado ampliamente cómo el modelo de ventajas comparativas tiene en sus bases la fuerza de trabajo barata, flexible y feminizada (Lara, 1998).

A lo anterior se añade para el caso particular de la producción de flores de corte, la concepción tradicional de que estas tareas son mucho más propias de lo femenino asociadas a valores como la habilidad con las manos, la delicadeza y el cuidado, lo que responde a una demanda que busca satisfacer una necesidad estética como lo es un ramo de flores (Lara, 1998).

Estas tendencias modernizadoras del sector agrícola encuentran un terreno laboral ideal en lo que se ha denominado feminización del mercado de trabajo, tal como afirma Standing $(1999,589)$ :

"La feminización es un concepto intencionalmente ambivalente, pues significa tanto: i) que se ha incrementado de manera significativa la participación de las mujeres en los mercados laborales simultáneamente con una caída relativa, cuando no absoluta, de la participación de los hombres; y ii) que los tipos de empleos que produce esta clase de apertura económica están asociados, correcta o incorrectamente (...) con el patrón histórico de participación de la fuerza de trabajo de las mujeres, patrón cuya característica principal es la flexibilización, que se expresa en los tipos de contratos, las formas de remuneración, los alcances y formas de seguridad social, y el acceso a la capacitación".

Como bien comenta Bossio (1990) los niños y las niñas representan una fuerza de trabajo perfectamente flexible para actividades que requieren pocos conocimientos y técnicas, por lo que en este tipo de ramas productivas el trabajo infantil se vincula a un conjunto de condiciones estructurales relacionadas con las tendencias a flexibilizar y precarizar los mercados laborales en aras de la obtención de ventajas competitivas mediante la disminución de costos productivos. Esto se profundiza con la presencia de las niñas jornaleras porque no sólo se flexibiliza el mercado de trabajo, también se feminiza en las dos ascepciones que indica Standing (1999).

Para la floricultura del sur del Estado de México, la feminización del mercado de trabajo representa la disponibilidad de trabajadoras dispuestas a aceptar condiciones flexibles a bajos salarios, lo que se traduce en un incremento de la competitividad, el comercio y la inversión en el sector. Incluso, se traduce en mayores incentivos fiscales por parte del Estado, bajo la premisa de que en la medida que los productores muestren mayor competitividad internacional entonces son objeto de incentivos estatales.

Lara (1998) afirma que para el caso de la floricultura estos procesos contribuyen a produndizar las desigualdades y la segmentación del mercado de trabajo por diversas vías tales como el género, la etnia y la edad, en este sentido contribuyen a la contratación de grupos sociales más vulnerables como las niñas jornaleras.

\section{Las niñas jornaleras en la floricultura: el género y la edad como dimensiones diferenciadores de las condiciones laborales}

El lugar que ocupa la región florícola en el sur del Estado de México en el sistema agrícola moderno y globalizado tiene sus efectos en las formas de organización y segmentación de los mercados laborales 
y en los sistemas de gestión de la fuerza de trabajo asalariada marcada por un importante proceso de feminización. Al respecto destaca la concepción empresarial extendida a nivel local sobre las ventajas de contratar más mujeres que hombres en las actividades propias del corte y embalaje de las flores. En palabras del Gerente de la Asociación Mexicana de Floricultores:

"Las mujeres son una ventaja, son mucho más responsables, no hacen san lunes, no se emborrachan, no se pelean y cuidan más su trabajo, tienen hijos y saben que tienen que mantenerlos, es difícil que falten al trabajo, muchas de ellas están muy interesadas en enseñar el oficio de la flor a sus hijos y sus hijas, (...) por eso mismo los floricultores las prefieren, y bueno no es lo mismo una mujer armando un ramo que un hombre, los hombres somos más toscos más brutos para menear las manos, en cambio las mujeres son más delicadas más cuidadosas".

Igualmente lo ilustran las propias trabajadoras de la flor quienes reconocen que el esquema del mercado de trabajo que les ofrece la floricultura ha cambiado pues ellas son igual o incluso más solicitadas que los hombres en los empleos que ofrecen los empresarios locales. Lo que se complementa con la necesidad de mayores ingresos familiares debido fundamentalmente a las crisis económicas recurrentes que impactan en las economías domésticas en el contexto rural mexicano:

“(...) pues qué le digo, con lo que gana el marido no se puede vivir, no hay para las tortillas no hay para los frijoles, y una como mujer debe buscarle y ayudarle al marido, por eso voy al corte de la flor, porque allá poco pero me gano algo y ya le ayudó a mi señor, (...) y somos hartas las que vamos al corte porque nos llaman a hartas, nos dan el trabajo, nos dejan llevar a los hijos $y$ unos pesos nos pagan, anterior a la flor solamente los señores eran los que iban al campo, uno le podía ir a ayudar a la pisca pero no era de pago, ahora si vamos hartas mujeres." [Mujer cortadora, 34 años]
De esta forma, es la convergencia de diversos factores, tales como el posicionamiento de la floricultura en el ramo de los cultivos de vanguardia, la percepción de mayor responsabilidad por parte de las mujeres, la atribución de cualidades de delicadeza y cuidado a las trabajadoras y la necesidad de las unidades domésticas de ampliar los ingresos monetarios, lo que ayuda a explicar, en términos generales, la gestión y organización de la fuerza de trabajo en el cultivo de las flores de corte bajo invernadero.

Lo anterior resulta de suma importancia para comprender las bases sobre las que se sustenta la organización y segmentación del mercado de trabajo en la floricultura, ya que los procesos de flexibilización y feminización se extienden también al trabajo de las niñas y los niños. Las bases sobre las que se fundamentan estos procesos tienen un vínculo directo con las ventajas que el sector empresarial local encuentra en esta modalidad y en el perfil de la fuerza de trabajo que está ampliamente disponible en la región, familias enteras, mujeres, niñas, niños y migrantes.

Con el auge de la floricultura en la zona del sur del Estado de México, las empresas floricultoras comienzan a demandar mano de obra, lo que deriva en un rápido proceso de salarización del trabajo agrícola en la floricultura de corte bajo invernadero. Desde un principio se trataba de mano de obra local integrada por las familias campesinas que al ver insuficiencia en sus ingresos con los cultivos tradicionales y la agricultura de subsistencia, se vieron obligados a vender su fuerza de trabajo en las empresas floricultoras.

Conjuntamente con el boom de la floricultura muchos pequeños productores cambiaron el giro productivo y apostaron por las flores de corte y el follaje, producidos mediante procesos y tecnologías rústicas y sin grandes inversiones. Los pequeños floricultores forman hoy en día el grueso de los productores de la región al tiempo que muchos de ellos y sus familias se emplean en las grandes agroindustrias floricultoras de exportación.

La actual floricultura bajo invernadero del sur del Estado de México se convirtió así en uno de los principales demandantes de mano de obra, al grado de 
que poco a poco han incrementado las migraciones provenientes de los estados del sur de México y de Centroamérica como un destino para emplearse en la floricultura ${ }^{1}$.

Así, los empresarios locales justifican la presencia de niñas jornaleras en términos de escasez de mano de obra en tiempo de fuerte producción, sin embargo, una mirada más integral del fenómeno obliga a considerar otra serie de factores relacionados con la flexibilidad y feminización de los mercados de trabajo. Las exigencias de los mercados globalizados resultan en una búsqueda de fuerza de trabajo con cualidades como la temporalidad y la maleabilidad que presentan los grupos más vulnerables.

A ello se suman diversas percepciones ampliamente extendidas entre el empresariado local sobre la presencia de niñas jornaleras en los campos y los invernaderos. En primer lugar, los floricultores locales mejor posicionados en el mercado se auto atribuyen la característica de la bondad para con las familias jornaleras pues les hacen un favor al emplear a las hijas, en las propias palabras de uno de ellos:

\section{“(...) uno les hace un favor de que puedan venir con las hijas, las chiquillas aprenden en el corte, y ayudan a la familia, la gen- te jodidona solo puede salir trabajando, si no es gente de trabajo no pueden vivir y aquí yo les doy una oportunidad para que se ganen la vida y aprendan y si cumplen y le echan ganas es bueno para nosotros." [Floricultor empresa A]}

Igualmente, otra justificación sobre el empleo de niñas tiene que ver con la dimensión y las cualidades por vía de la edad. Para los floricultores los niños y las niñas por ser pequeños están más cerca del suelo y se tardan menos tiempo en cortar las flores y limpiar el terreno, lo que se traduce en mayor eficiencia en términos de tiempos. El testimonio del Gerente de la Asociación Mexicana de Floricultores es muy claro al respecto:

"-¿Hay niños empleados en la floricultura? mira la declaratoria de los derechos de los niños te limita de alguna manera, sin embargo hay tareas, o sea, hay actividades que debieran ser exclusivas de los niños. - ¿Cómo cuáles? la desbotonada por ejemplo, es el trabajo más pesado que se tiene en la floricultura, es, cada vez que tú ves una planta tienes que hacerle la reverencia, desde arriba hasta abajo quitarle todos los botones secundarios, pero como tú estás a cierto nivel y más cuando es, el producto, el surco está alto o está en ladera, cuando está en ladera no tienes problema porque tienes la planta aquí no más te agachas así, pero cuando está hasta abajo en plano en tierra plana, es empezar a desbotonar.

- ¿Se requiere mucho esfuerzo? Sí, son dos reverencias por cada tallo, y es muy cansado, cuando terminas una cama, que le llaman, si no te echas una maroma ya no aguantas, te queda, hay veces que te quedas así de lo cansado que es, para los niños eso es el ideal, por qué, porque para los niños por su estatura empezarían y no tendrían que agacharse y es un trabajo muy fácil que podría ser de niños, no soy partidario de que se explote a los niños desde pequeños, pero sí de que se les enseñe a hacer las cosas y al final de cuentas de acuerdo a sus habilidades y sus destrezas $y$ sus fortalezas ellos realicen lo que les es posible, se les ayuda a ellos y el productor ve mejoras en sus resultados, por qué, con estas habilidades así de fácil, los niños son mucho más eficientes".

Las tareas que exige la producción de flores son variadas, por ejemplo sembrar, que consiste en ir

1 Al respecto cabe señalar que dada la estacionalidad de este tipo de productos de vanguardia, con notables picos productivos durante el año, las familias jornaleras migrantes inician su recorrido por el territorio mexicano en busca de empleos temporales, una vez que termina el corte de la flor siguen hacia los estados del norte a las cosechas de las hortalizas y frutas. Muchas de estas familias jornaleras migrantes carecen de documentación legal lo que las expone directamente a una situación laboral precaria, vulnerable e incierta (Pérez, 2005). 
enterrando los bulbos en las camas para que posteriormente crezcan las flores; otra actividad es limpiar, desyerbar, que consiste en retirar las yerbas y maleza que va creciendo alrededor de las flores y que puede ser dañina o aumentar el riesgo de propagación de plagas. Tanto sembrar como desyerbar son actividades que exigen al trabajador posiciones del cuerpo tales como permanecer agachados, hincados, o en cuclillas con el objeto de agilizar las actividades. De ahí que se prefiere reservar esas tareas para las niñas, debido al argumento generalizado respecto a que por su tamaño y edad se le facilita mucho más movilizarse por el suelo, por lo que se logra dar mayor eficiencia y rapidez a las actividades.

Este argumento resultó muy común durante la recogida de información, sobre todo para los empleadores y encargados, paradójicamente, al preguntarles a las niñas sobre los malestares más frecuentes que sentían al realizar actividades en la floricultura, los dolores por permanecer agachadas y doblar el cuerpo fueron una constante en la mayoría de los testimonios de las niñas. Incluso en los dibujos fue también una constante mostrar que estar hincadas $y$ por el suelo es una de las principales posiciones corporales que utilizan al desempeñar las actividades (Ilustración 1).

\section{Ilustración $\mathbf{1}^{2}$}

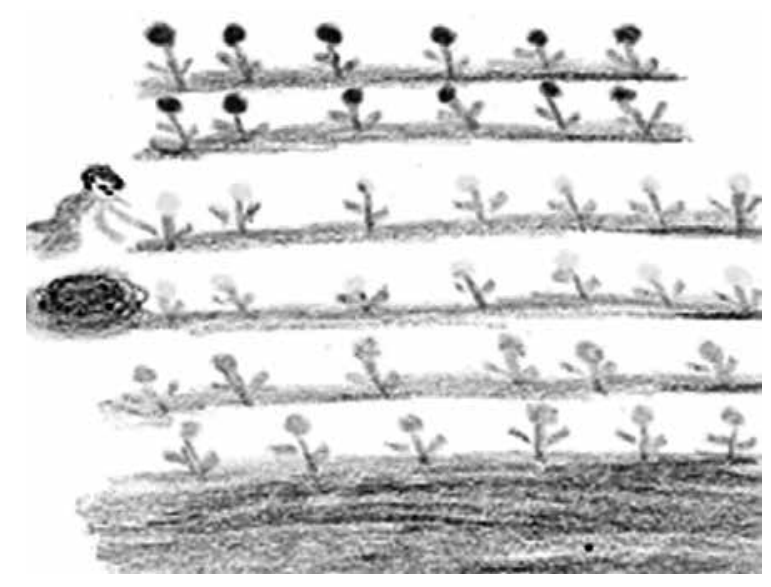

2 Fuente: Niña jornalera, 9 años, Grupo de Discusión 6.
Otro argumento que justifica el uso específico de niñas jornaleras en las temporadas de alta demanda de trabajadores en la floricultura es su característica de mayor disciplina. Según las palabras de un encargado de personal en los invernaderos: "los muchachitos son groseros y a veces están en malos pasos, toman o se meten drogas, pero las niñas por ser mujercitas son más responsables, no pelean con otras, les interesa el trabajo" [Encargado empresa A]. En este sentido, para este contexto laboral, las niñas cumplen con las características de menor conflictividad.

Durante el trabajo de campo fue posible observar ciertas prácticas respecto del trato y la forma en que se dan órdenes a los empleados incluyendo a las niñas jornaleras. Dado que en "la producción" es una época de alta demanda, se requiere de rapidez y funcionamiento eficaz de los empleados ya que los pedidos son constantes y pueden llegar a ser tan sorpresivos que algunos de ellos se exigen de un momento a otro. Así, bajo el argumento de una fuerte presión de la demanda de flores, se exige rapidez y eficiencia a los empleados.

El papel del "encargado" es clave en este proceso. Es éste quien hace rondas para observar y verificar el trabajo que se realiza, organiza a los empleados y asigna actividades; en general los empleados están bajo su subordinación. Para los empleados adultos, escuchar las órdenes y amenazas del "encargado" resulta en una experiencia desagradable que les infunde cierto temor de perder el trabajo en cualquier momento. Las niñas por lo general expresaron sentirse amenazadas, angustiadas y asustadas con los gritos e insultos que les profiere el encargado. Uno de los principales miedos en el trabajo que fue reconocido abiertamente por las niñas jornaleras fueron los gritos y regaños de los patrones:

“(...) -a mí lo que no me gusta es que los patrones luego son como enojones y regañones, y no más que te ven un poco recargada en la mesa y ya te gritan y no me gusta que los niños me avientan la basura y el encargado ni les dice nada pero bien que 
conmigo es bien estricto." [Niña, 9 años, Grupo de discusión 3]

Igualmente llama la atención que la característica de aguantar el sufrimiento es atribuida a las niñas como un aspecto positivo para el trabajo. Según un encargado de un invernadero las niñas aguantan cualquier condición adversa. La siguiente conversación pone en evidencia este aspecto:

“-¿Por qué transportan a las niñas en el mismo lugar que la gasolina y los fumigantes?

- Mire aquí de lo que se trata es de trabajar $y$ uno como encargado tiene que ver que todo se organice como se debe, y uno organiza el transporte de la gente y las niñas van ahí porque ellas son buenas para el trote, son buenas para el sufrir, les gusta el trabajo y lo cumplen, en cambio los jovencitos no son tan obedientes." [Encargado empresa A]

Evidentemente, a las niñas al igual que a las mujeres se les atribuyen habilidades especiales por la vía del género, los empresarios locales y los encargados del personal consideran que las niñas jornaleras son delicadas y curiosas, tienen mucho más cuidado y ponen más empeño en el empaque y la formación de arreglos florales.

“(...) - la verdad no voy a meter a un joven o a un niño en el empaque o no lo voy a poner a armar ramos porque no queda, como que no queda, eso es un trabajo de mujeres, y eso se ve en el resultado porque las mujeres y las niñas saben tratar la flor, y para los jóvenes y los niños hay otras cosas que hacer, hay que cargar, hay que limpiar, hay que fumigar, eso de los ramos ni para qué ponerlos ahí, se vuelve un desorden para qué quiere." [Encargado empresa A]

Varias prácticas observadas durante el trabajo de campo en las empresas florícolas subyacen en la división sexual del trabajo y permean la participación de las niñas jornaleras, por ejemplo se habla de tareas más pesadas para los hombres que para las mujeres, pero en el día a día las mujeres y las niñas realizan todo tipo de actividades incluyendo las que se etiquetan como típicamente masculinas.

En la fase del empaque por ejemplo, cargar y apilar las cajas o los ramos es una labor que en el discurso de los empleadores y encargados se vislumbra como una actividad propia de varones, niños y adolescentes, no obstante son tareas que se realizan también por las niñas. Este proceso requiere de suficiente fuerza para soportar pesos aproximados a 10 kilogramos en cada recorrido de la salida de la empresa hacia el camión que las transportará. Ello se lee con claridad en el siguiente testimonio de una niña de 10 años que se emplea en la floricultura:

“-¿Cuáles son las actividades que más te cuesta hacer?

- Las de cargar, no me gusta mucho, es bien cansado, además hay que saber poner bien las flores, si no las pones bien se caen, si no las agarras fuerte se te pueden caer, eso no me gusta, yo cargo como cinco cajas grandes en un viaje pero otras niñas cargan de a siete cajas yo mejor nomás cargo cinco." [Niña, 10 años, Grupo de Discusión 6]

En el mismo sentido, las actividades relacionadas con la aplicación de fungicidas y productos químicos para evitar las plagas en las flores se describe en el discurso como propias del género masculino, pero los testimonios y las ilustraciones (Ilustración 2) que realizaron las niñas muestra que ellas son también incorporadas en estas tareas.

“-A mí no me gusta lo de fumigar. -¿Por qué?

-Es que hay que jalar la manguera y entonces dijeron que era necesario fumigar, yo tenía que cargar la manguera y nos dicen algunos disparates porque no jalo la manguera.

-¿Quién dijo los disparates?

-El patrón." [Niña, 9 años, Grupo de Discusión 1] 


\section{Ilustración $2^{2}$}

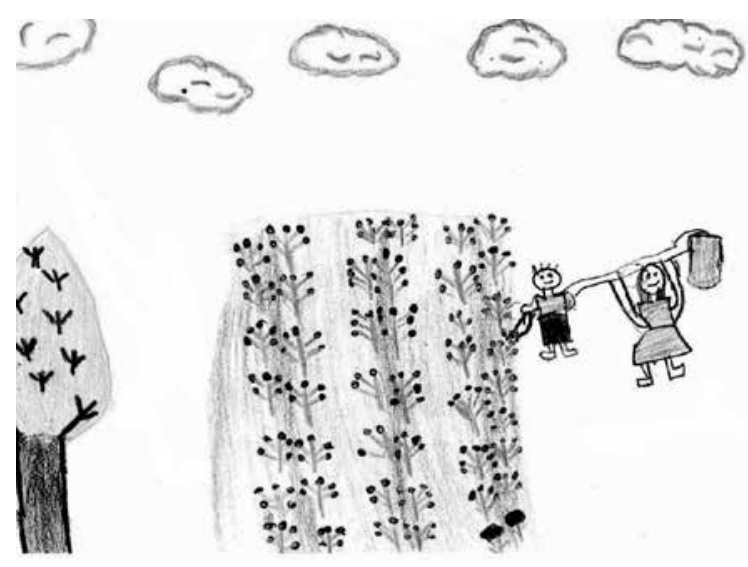

A criterio de los empleadores y encargados pareciera ser que en el área de empaque y embalaje no es muy grande la demanda de esfuerzo físico requerido, no obstante, las niñas expresan que se cansan al mantenerse de pie todo el día, les duelen los pies y la cabeza. A veces les desagrada el olor de "los fumigos" que se concentra en dicho espacio.

"Cuando empacas te duele mucho la cabeza no puedes pensar en contar los tallos ni en nada de eso, y el dolor de cabeza, el dolor de los pies, porque tienes que estar parada." [Niña, 11 años, Grupo de Discusión 3]

Una parte importante durante las jornadas laborales son los tiempos libres y los descansos. Durante las faenas dedicadas a la producción de flores, los empleados tienen una hora libre destinada para el almuerzo. Los trabajadores deben llevar su propia comida o bien salir a comprar algo. Se observó una práctica recurrente durante el tiempo del almuerzo. Los niños y adolescentes varones aprovechan para jugar y distraerse en las instalaciones de la empresa. Jugar con los patines ${ }^{3}$ fue uno de los pasatiempos más mencionados entre los niños, igualmente se ob- servó que los niños y adolescente varones aprovechaban el almuerzo para jugar "cascaritas" de futbol, carreras y correteadas.

Sin embargo, la hora del almuerzo no representa una oportunidad de descanso y diversión para las niñas. Ellas deben preparar y servir los alimentos que llevan para la familia. Preparan los tacos o tortas, sirven el agua y al final limpian el espacio que ocuparon ellas y sus familias para almorzar. Muchas expresaron que les parecía injusto no poder jugar en la hora del almuerzo ya que veían cómo se divertían los hermanos y otros niños varones.

Al respecto es importante señalar que el trabajo doméstico y de cuidados que llevan a cabo las niñas y las adolescentes es crucial en la reproducción económica y social. Son ellas quienes relevan a las madres, abuelas u otras mujeres adultas en el trabajo doméstico y en las actividades de cuidados a terceros cuando éstas salen al mercado laboral extra doméstico. Tradicionalmente se asume que las tareas de cuidado son típicamente femeninas y se les ha asignado a las mujeres la responsabilidad total en la crianza de niños y niñas, incluso cuando quienes realizan dichas tareas se encuentran en cortas edades, desde la niñez y la adolescencia.

La literatura especializada ha encontrado que las niñas y adolescentes además de realizar actividades escolares, colaboran en las tareas domésticas, participan en los cuidados de los miembros más pequeños del hogar y en algunos casos salen también al mercado de trabajo (Hoyos, 2000).

De este modo, la participación de niñas jornaleras en la floricultura de corte bajo invernadero es el resultado de varios aspectos que se entrelazan y que resultan en la reproducción de patrones de inseguridad, inestabilidad y precariedad laboral. Este escenario contribuye a que las niñas jornaleras se consoliden poco a poco como un grupo que tempranamente se

Fuente: Niña, 11 años, Grupo de Discusión 1.

El patín, es un artefacto para transportar cargas pesadas, está conformado por tablas de madera y un soporte de ruedas, es sumamente demandado entre los niños, sobre todos los patines que cuentan con sistema de elevador para subir y bajar la carga. Un niño empuja a quienes se atreven a subirse sobre la plataforma del patín y estos son llevados a lo largo de las instalaciones o por terrenos inclinados. 
entrena para especializarse en las destrezas que requiere un mercado de trabajo globalizado, y a su vez se entrenan para cumplir con los mandatos de disciplina y sumisión, lo que al mismo tiempo se traduce en la configuración de mercado de trabajo con vigorosas asimetrías de género, atributo necesario para la consolidación de una rama productiva moderna y de exportación.

\section{Consideraciones finales}

A la luz de la descripción realizada resulta necesario retomar la idea apuntada al principio sobre el vínculo específico que se establece en la floricultura del sur del Estado de México como sector que ha sufrido transformaciones productivas para adecuarse a los requerimientos globales y la forma de gestionar la mano de obra y a partir de ello, establecer algunas conclusiones finales.

En primer lugar, los resultados obtenidos en la investigación permiten confrontar el discurso mayoritario que tiende a justificar la presencia de niños y niñas como trabajadores basado en una actitud de bondad que beneficia por igual a empresarios y familias campesinas y jornaleras.

En este discurso generalizado subyace el interés por posicionarse en el mercado global, proceso que requiere de una mano de obra flexible, barata y disponible. El discurso sobre el bondadoso empresariado local y la escasez de fuerza de trabajo en los picos productivos, de cierto modo oculta las desigualdades por las vías del género y la edad que se establecen en este mercado de trabajo.

La marcada presencia de niñas jornaleras en la floricultura ilustra el carácter discriminatorio que adquiere la forma de gestionar la mano de obra en esta rama productiva. Al parecer hay un nexo importante entre la práctica del trabajo infantil y la flexibilidad, precariedad y feminización del trabajo en la floricultura de corte, lo que a su vez funciona como un modelo que reproduce esquemas de segmentación por género y por edad. Ello dista mucho de garantizar los derechos más fundamentales de uno de los sectores más vulnerables de la infancia en México, las niñas jornaleras.
En este mismo sentido, el discurso sobre las habilidades femeninas que se requieren en este tipo de cultivo delicado, pone en evidencia un modelo laboral basado en la diferenciación de género, colocando a las mujeres y las niñas en una categoría de trabajadoras con opciones restringidas en cuanto a sus derechos laborales y de movilidad social.

Como se vio en los resultados del trabajo de campo, los discursos se contraponen con la práctica cotidiana. Ello se observa con claridad en la concepción generalizada de tareas masculinas y femeninas y en la realización de ambas por parte de las mujeres y las niñas. Asimismo, se observó lo que se conoce ampliamente como la doble jornada, en la que las niñas jornaleras además de realizar labores propias de la floricultura realizan actividades de cuidado sin oportunidades de descanso y recreación como lo hacen los varones.

La precariedad laboral que se observa en este mercado de trabajo local impacta en el grueso de los trabajadores asalariado que ahí se emplean, pero para las niñas jornaleras hay una doble segmentación que se da por la dimensión de la edad y del género. Las condiciones laborales observadas no sólo hacen posible el logro de la competitividad económica sino que ayudan a perpetuar y reproducir esquemas laborales desiguales.

En definitiva, el modelo de gestión flexible de la fuerza de trabajo que incluye la presencia de niños y niñas como trabajadores asalariados representa una oportunidad para garantizar el volumen de mano de obra a bajo costo necesaria en los picos productivos. La temporalidad, la flexibilidad y la disponibilidad que ofrecen las niñas jornaleras es un elemento clave que garantiza la viabilidad de este cultivo y su capacidad para hacer frente a la competitividad de los mercados agrícolas globalizados.

Al respecto es posible afirmar que en la organización de la fuerza de trabajo de la floricultura conviven antiguos estereotipos tradicionales en el sector agrícola, tales como el control arbitrario y los castigos e insultos a los trabajadores, con nuevas estrategias de organización en las que los cambios inesperados en las exigencias del mercado global exigen una fuerza de 
trabajo feminizada y flexible que fácilmente se adapte a los cambios que se presentan. En este sentido el trabajo de las niñas jornaleras no tiene ventajas únicamente por el bajo costo económico que les representa a los empleadores, sino que es parte de una fuerza de trabajo con características de sumisión y obediencia que resultan en mayor eficiencia productiva para las empresas.

Asimismo, los procesos descritos son trascendentales en el análisis de la participación de las niñas jornaleras, pues si se toma en cuenta el contexto de informalidad y arbitrariedad que caracteriza la gestión y organización de la fuerza de trabajo, fuera de cualquier marco regulador de protección laboral, entonces cobra sentido la presencia constante las niñas en este mercado de trabajo, en donde se favorece el aprovechamiento de una mano de obra fácilmente moldeada a las condiciones de disponibilidad horaria, escasa calificación y bajos niveles salariales.

En este contexto, es importante señalar que la aportación que hacen las niñas a la floricultura además de ser invisibilizada es infravalorada bajo el argumento de que sólo realizan actividades delicadas y marginales de menor envergadura en comparación con el trabajo de los varones y los adultos.

La infra-valorización de la aportación económica que hacen estas niñas a la producción florícola es importante en el sentido de que realizan las actividades cuyo costo no es pagado en su totalidad por los empleadores. Barrer, limpiar, desyerbar, cargar, son actividades vistas como fáciles y marginales que al ser efectuadas por las niñas funcionan como un importante minimizador de costos para los empleadores y en muchos de los casos una importante entrada de ingresos a la economía familiar.

Una reflexión obligada en cuanto a la participación laboral de las niñas en la floricultura es el papel del Estado como garante de los derechos de los niños y las niñas en este contexto local, el Estado omite su responsabilidad pues no garantiza que se cumpla la legislación establecida en material de trabajo infantil y además refuerza esta práctica al promover su política de ayuda crediticia a todos aquellos productores que comprueben competitividad a nivel internacio- nal y posibilidades de generación de divisas, lo que constituye un mecanismo que refuerza la utilización de esquemas laborales flexibles y feminizados.

Estudiar el trabajo de las niñas en una rama productiva como las flores, resulta interesante tanto en su calidad de mercancía como por su carácter de metáfora. El consumo de bienes suntuosos con valor estético como las flores es un fenómeno estructurado bajo una compleja interacción de fuerzas económicas y sociales sustentadas en la interconexión entre las dinámicas laborales y las dinámicas domésticas que ponen en el centro a la práctica y reproducción de roles de género que resultan en notables asimetrías sociales.

Hacer visibles a las niñas jornaleras resulta indispensable para dilucidar la contribución de las trabajadoras del campo desde tempranas edades a la economía nacional, igualmente es necesario visiblizarlas para orientar las políticas públicas, tomando en cuenta los procesos de reproducción de desigualdades.

Un nuevo abordaje en la política pública que haga visible a estos grupos sociales y reconozca que las transformaciones agrarias recientes han deteriorado las condiciones laborales que ya de por si eran precarias para los trabajadores agrícolas, pero además que ponga atención en los procesos que reproducen los roles de género desde tempranas edades. Solo así, el Estado y la sociedad podrán pagar la deuda histórica que se tiene con estas niñas.

\section{Referencias}

Aguado, E. (1998). Una mirada al reparto agrario en el Estado de México, 1915-1992: de la dotación y restitución a la privatización de la propiedad social. El Colegio Mexiquense. México.

BANCOMEXT (2001). Guía de exportación sectorial, flores y plantas. México: Banco Nacional de Comercio Exterior.

Bossio, J. (1990). La OIT y el trabajo infantil: una perspectiva Latinoamericana. OIT, Lima Perú.

Castro, P. (2003). Chayotes, burros y machetes. El Colegio Mexiquense, A.C., Zinacantepec - México.

CONEVAL, (2012). Informe de pobreza en México 2010: el país, los estados y sus municipios. Consejo Nacional de Evaluación de la Política de Desarrollo Social, México.

Hoyos, S. (2000). ¿Y quién la mandó a ser niña? El trabajo infantil doméstico desde una perspectiva de género. En: Acosta, G., García, E. \& Hoyos, S. (coords.) Trabajo infantil doméstico ¿Y 
quién la mandó a ser niña? Bogotá, Fondo de las Naciones Unidas para la Infancia (UNICEF) / TM Editores.

INEGI (2012). Encuesta Nacional de Ocupación y Empleo 2011, Módulo de Trabajo Infantil. México.

Lara, S. (1998). Nuevas experiencias productivas y nuevas formas de organización del trabajo en la agricultura mexicana. Procuraduría Agraria, México.

Lezcano, A. (2002). El trabajo infantil en algunos centros de concentración urbano. Perfiles y estrategias laborales de supervivencia. En: Bialakowsky, A., Lezcano, A. \& Senén, C. (comp.) Unidad en la diversidad. Estudios laborales en los 90. Facultad de Ciencias Sociales UBA, Buenos Aires.

Morett, J. \& Cosío, C. (2004). Los jornaleros agrícolasen México. Universidad Autónoma Chapingo, Diana, México.

Orozco, M. (2007). Entre la competitividad local y la competitividad global: floricultura comercial en el Estado de México. En: Convergencia, Revista de Ciencias Sociales, (45): 111-160.

Pérez, E. (2005). Reclutamiento de migrantes en la región floricultora del sur del Estado de México. Tesis para la obtención del grado de maestro en Ciencias Sociales con especialidad en Desarrollo Municipal. El Colegio Mexiquense A.C. Zinacantepec, México.

Reigada, A. (2011). Reestructuración agraria, migración laboral y feminización del trabajo en Andalucía. En: Agricultura, Sociedad y Desarrollo, Enero - Abril. España.

Revista Expansión (1998). La floricultura bajo invernadero. En: Revista Expansión, 12 (agosto): 307-316. México.

Rubio, B. (2002). Reestructuración agroindustrial, nuevas pautas de explotación rural y movimiento de productores en México durante los año noventa. En: Rubio, B. \& Martínez, C. (comp.) Reestructuración productiva, comercialización y reorganización de la fuerza de trabajo agrícola en América Latina. Plaza y Valdez, México.

Seffó, J. (2005). La calidad es nuestra, la intoxicación... ide usted! Atribución de la responsabilidad en las intoxicaciones por plaguicidas agrícolas, Zamora, Michoacán, 1997-2000. El Colegio de Michoacán, México.

Standing, G. (1999). Global feminization through flexible labor: a theme revisited. En: World Development, International Labour Organisation, 27(3): 583-602. Geneva - Switzerland. 